\title{
Positron Kinetics in Soft Condensed Matter
}

\author{
R. D. White ${ }^{1, *}$ and R.E. Robson ${ }^{1,2}$ \\ ${ }^{1}$ ARC Centre for Antimatter-Matter Studies, James Cook University, Townsville 4810, Australia \\ ${ }^{2}$ ARC Centre for Antimatter-Matter Studies, Australian National University, Canberra 2600, Australia
}

(Received 22 March 2009; revised manuscript received 30 April 2009; published 9 June 2009)

We outline a new kinetic theory for positrons in soft matter, which blends together cross sections for positrons scattering from single molecules, with the structure function of the medium as a whole. Numerical results are presented for positrons in liquid argon, where negative differential conductivity arises from both positron formation and the structure of the medium.

DOI: 10.1103/PhysRevLett.102.230602

PACS numbers: 05.20.Dd, 34.80.Uv, 36.10.Dr

Introduction.-An understanding of the interaction of free positrons with soft, biological matter is important for medical diagnostic procedures, such as positron emission tomography [1]. However, a deficiency in the understanding of the fundamental physics at the atomic and molecular level limits the full potential of these important applications, something which has prompted a renewed effort in both measurement [2] and ab initio theoretical calculation $[3,4]$ of positron-atom and molecule scattering cross sections including biomolecules [5]. While we expect full sets of cross sections for the relevant biological molecules to become available eventually, such microscopic information is by itself not sufficient for applications to the macroscopic world. Although there has been renewed interest in the transport of positrons in gaseous systems [6,7], this Letter addresses for the first time the next step on the way to dealing with these important applications, namely, the calculation of transport coefficients for positrons in soft condensed matter. As is the case for electrons in liquids (see the review [8]), the microscopic-macroscopic link is provided by kinetic theory, with Boltzmann's kinetic equation being modified to account for structure and coherent elastic scattering. This Letter first outlines the essential theory for positron transport in structured media including a multiterm solution technique which avoids the limitations of the two-term approximation traditionally employed for electrons. Second, we consider the case of positrons in liquid argon, focusing, in particular, on the effects of positronium (Ps) formation and liquid structure, and their influence on transport coefficient duality and negative differential conductivity (NDC) - the fall of the drift velocity with increasing reduced electric field $E / n_{0}$.

Positron Boltzmann equation.-For sparse positrons of charge $e$, mass $m$, and velocity $\boldsymbol{c}$ in a gaseous or condensed matter medium subject to an electric field $E$, one solves the Boltzmann kinetic equation for the positron phase distribution function $f(\boldsymbol{r}, \boldsymbol{c}, t)$ :

$$
\left(\frac{\partial}{\partial t}+\boldsymbol{c} \cdot \nabla+\frac{e \boldsymbol{E}}{m} \cdot \frac{\partial}{\partial \boldsymbol{c}}\right) f=-J(f),
$$

where $J=J_{\text {elas }}+J_{\text {inel }}+J_{\mathrm{Ps}}+J_{a}$ is a linear collision op- erator representing the various collisional processes with the medium. The two most important processes are the elastic collision operator $J_{\text {elas }}$, modified from Boltzmann's original expression to account for coherent scattering from structured media, using a generalization of [9] (see below) and the operator describing Ps formation, $J_{\mathrm{Ps}}=n_{0} c \sigma_{\mathrm{Ps}}(c)$, where $n_{0}$ is the number density of the molecules of the background medium and $\sigma_{\mathrm{Ps}}(c)$ is the Ps-formation cross section. The positron annihilation operator $J_{a}$ is similarly defined in terms of an annihilation cross section $\sigma_{a}(c)$, while $J_{\text {inel }}$ is taken here to be the semiclassical inelastic collision operator $[8,10]$. This is the microscopic picture. Macroscopic properties are then obtained as averages of certain quantities $\phi(c)$ over positron velocities $\langle\phi(c)\rangle=$ $\frac{1}{n} \int d \boldsymbol{c} f(\boldsymbol{r}, \boldsymbol{c}, t) \phi(\boldsymbol{c})$, where $n(\boldsymbol{r}, t)=\int d \boldsymbol{c} f(\boldsymbol{r}, \boldsymbol{c}, t)$ is the positron number density, e.g., the mean energy $\varepsilon \equiv$ $\left\langle 1 / 2 m c^{2}\right\rangle$ and mean velocity $\boldsymbol{v} \equiv\langle\boldsymbol{c}\rangle$.

Spherical harmonics representation.-The first step in the solution of (1) is the spherical harmonics expansion

$$
f(\boldsymbol{r}, \boldsymbol{c}, t) \approx \sum_{l=0}^{l_{\max }} \sum_{m=-l}^{l} f_{m}^{(l)}(\boldsymbol{r}, c, t) Y_{m}^{[l]}(\hat{\boldsymbol{c}})
$$

where truncation at $l=l_{\max }$ is required for solution. The traditional "two-term" approximation $\left(l_{\max }=1\right)$ continues to be used exclusively in electron-liquid transport calculations [8], although it is known from gaseous electronics that this can lead to a serious error [11]. In best practice, the integer $l_{\max }$ is incremented until a prescribed accuracy criterion is met as considered here. Combining (1) and (2) leads to the following hierarchy of coupled integro-differential equations for $f_{m}^{(l)}$ :

$$
\begin{aligned}
\partial_{t} f_{m}^{(l)}+ & \sum_{l^{\prime} m^{\prime}}\langle l m| \boldsymbol{c} \cdot \nabla \\
& +\frac{e \boldsymbol{E}}{m} \cdot \frac{\partial}{\partial \boldsymbol{c}}\left|l^{\prime} m^{\prime}\right\rangle f_{m^{\prime}}^{\left(l^{\prime}\right)}=-\sum_{l^{\prime} m^{\prime}}\left\langle l m|J| l^{\prime} m^{\prime}\right\rangle f_{m^{\prime}}^{\left(l^{\prime}\right)}
\end{aligned}
$$

Expressions for the matrix elements of the streaming operator on the left-hand side are given in $[11,12]$. The collision matrices $\left\langle\operatorname{lm}|J| l^{\prime} m^{\prime}\right\rangle=\left[J_{\text {elas }}^{l}+J_{\text {inel }}^{l}+J_{\mathrm{Ps}}^{l}+\right.$ 
$\left.J_{a}^{l}\right] \delta_{l^{\prime}, l} \delta_{m^{\prime}, m}$ are all diagonal in $l$ and $m$, since the collision operators are all scalars.

Collision operators.-For elastic collisions of positrons with the constituent molecules of mass $m_{0}$ of either a gaseous or condensed medium at a temperature $T_{0}$ and number density $n_{0}$, the same operator applies for $l=0$ [8,9], namely, to $O\left(\left(\mathrm{~m} / \mathrm{m}_{0}\right)^{2}\right)$

$$
J_{\text {elas }}^{0}\left(f_{0}^{(0)}\right)=-n_{0} \frac{m}{m_{0} c^{2}} \frac{\partial}{\partial c}\left[c^{4} \sigma_{m}\left(f_{0}^{(0)}+\frac{k_{B} T_{0}}{m c} \frac{\partial f_{0}^{(0)}}{\partial c}\right)\right],
$$

where $\sigma_{m}=\sigma_{0}-\sigma_{1}$ is the momentum transfer cross section, $\quad \sigma_{l}=2 \pi \int_{0}^{\pi} d \chi \sin \chi\left[1-P_{l}(\cos \chi)\right] \sigma(c, \chi)$ are the partial cross sections, and $\sigma(c, \chi)$ is the differential scattering cross section. While the structure of the medium plays no role for $l=0$, for $l \geq 1, J_{\text {elas }}^{l}=n_{0} c\left[\Sigma_{0}(c)-\right.$ $\left.\Sigma_{l}(c)\right]+O\left(m / m_{0}\right)$ involves the static structure function $S$ [13] through the structure-modified partial cross sections,

$$
\Sigma_{l}(c)=2 \pi \int_{0}^{\pi} d \chi \sin \chi P_{l}(\cos \chi) \Sigma(c, \chi),
$$

where $\Sigma(c, \chi) \equiv S\left[\frac{2 m c}{\hbar} \sin \left(\frac{\chi}{2}\right)\right] \sigma(c, \chi)$ defines a differential cross section modified to account for coherent scattering. In the dilute gas phase limit, $S \rightarrow 1$, and it then follows that $\Sigma(c, \chi) \rightarrow \sigma(c, \chi)$. The two sets of partial cross sections are linked by the relationship

$$
\begin{aligned}
\Sigma_{l}(c)= & \frac{1}{4 \pi} \sum_{\lambda^{\prime} \lambda^{\prime \prime}} \frac{\left(2 \lambda^{\prime}+1\right)\left(2 \lambda^{\prime \prime}+1\right)}{2 l+1} \\
& \times\left(\lambda^{\prime} 0 \lambda^{\prime \prime} 0 \mid l 0\right)^{2} \sigma_{\lambda^{\prime}}(c) s_{\lambda^{\prime \prime}}(c),
\end{aligned}
$$

where $\left(\lambda^{\prime} 0 \lambda^{\prime \prime} 0 \mid l 0\right)$ is a Clebsch-Gordan coefficient and $s_{l}(c)=\frac{1}{2} \int_{-1}^{1} S\left[\frac{2 m c}{\hbar} \sin \left(\frac{\chi}{2}\right)\right] P_{l}(\cos \chi) d(\cos \chi)$. For Ps formation, the expression $J_{\mathrm{Ps}}^{l}=n_{0} c \sigma_{\mathrm{Ps}}(c)$ holds for all $l$, and similarly for annihilation. Matrix elements of the inelastic collision operator are detailed in [11]. Before proceeding with the numerical study, we note the following points: (i) For $s$-wave scattering, $\sigma(c, \chi)$ is independent of angle $\chi$, (6) reduces to $\Sigma_{l}(c)=\sigma_{0}(c) s_{l}(c)$, and the structure-modified "momentum transfer cross section" is then given by $\Sigma_{m}=\Sigma_{0}-\Sigma_{1}=\sigma_{0}\left(s_{0}-s_{1}\right)$. (ii) In the two-term approximation, only the low order "moment" $s_{0}-s_{1}=\frac{1}{2} \int_{-1}^{1} S\left[\frac{2 m c}{\hbar} \sin \left(\frac{\chi}{2}\right)\right](1-\cos \chi) d(\cos \chi)$ of $S$ is sampled [8]. On the other hand, a multiterm solution allows sampling of higher order moments of $S$ and hence gives more detailed information about the liquid structure. Beyond this, soft matter transport coefficients can be obtained immediately by application of the same analytical and numerical techniques employed for gaseous media.

Comments on the work of Cohen and Lekner and others.-The seminal work of [9] for electron transport in structured media and subsequent applications for electrons (see review [8]) and positrons [14] all make the two-term approximation, thereby losing accuracy in the representation of the velocity dependence of $f$ and reducing information on the structure of the medium. This Letter shows how these theories can be generalized by lifting these restrictions. Furthermore, the results presented below demonstrate that the Ps-formation rate must be calculated selfconsistently and not as a perturbation, as in [14].

Calculation and duality of transport coefficients.Differences exist between hydrodynamic transport coefficients defined using a flux-gradient relation (flux coefficients) and those defined using the diffusion equation (bulk coefficients) when nonconservative collisional processes are operative [15]. Of particular interest here is the duality in the drift velocities - bulk and flux drift velocities. The flux drift velocity $W_{F}$ is the mean velocity of positrons, while the bulk drift velocity $W$ can be thought of as the drift velocity of the center of mass in a time-of-flight experiment. When nonconservative processes, such as Ps formation, act to modify the center of mass, $W_{F} \neq W$. The path to obtaining hydrodynamic transport coefficients in gases is now textbook material [16], and suffice it to say that a further decomposition of $f_{m}^{(l)}(\boldsymbol{r}, c, t)$ is made in terms of gradients of density $\nabla^{j} n(\boldsymbol{r}, t)(j=0,1,2, \ldots)$. These quantities can also be represented as spherical tensors, generating a combined spherical harmonic-density gradient expansion. The resulting hierarchy of equations appears in $[11,12]$.

Positrons in liquid argon.-Any transport calculation requires complete sets of single scattering positron impact cross sections, e.g., for Ar [6]. In this set, all cross sections are assumed isotropic and annihilation is neglected. These cross sections and the structure-modified elastic cross sections (6) are displayed in Fig. 1. The structure factor for liquid $\mathrm{Ar}$ at $85 \mathrm{~K}$ (atomic density is $0.02125 \AA^{-3}$ ) is taken from [17], while in the dilute gaseous limit, $S$ is set to unity. We note that the influence of structure is reduced at higher energies since de Broglie wavelengths, and hence coherent scattering effects, are reduced and the liquid phase elastic cross sections approach those in the dilute gas phase limit. It follows that the liquid positron transport properties approach those of the dilute gaseous phase in the high

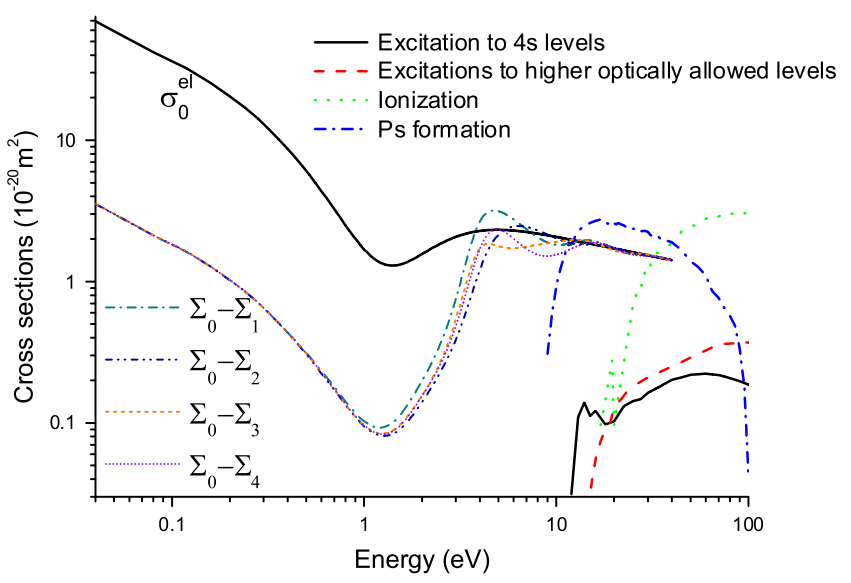

FIG. 1 (color online). Positron impact cross sections in dilute gaseous $\operatorname{Ar} \sigma_{i}$ (see [6] and references therein) and structuremodified elastic cross sections $\Sigma_{0}-\Sigma_{l}$ in liquid $\mathrm{Ar}$ at $85 \mathrm{~K}$. 
field, high energy limit. In Figs. 2 and 3, we display transport properties for positrons in liquid and dilute gaseous $\mathrm{Ar}$ at $85 \mathrm{~K}$ as a function of the reduced electric field $E / n_{0} \quad\left(1\right.$ townsend $\left.=1 \mathrm{Td}=10^{-21} \mathrm{~V} \mathrm{~m}^{2}\right) . \quad$ Excellent agreement exists between the current results for the dilute gaseous case and those of independent Monte Carlo results recently reported [6]. We present converged multiterm results only in these figures but note that two-term results are generally accurate to within $1 \%$ for the particular case and transport coefficients under consideration.

In Fig. 2, we display the mean energy and Ps-formation rate. At low $E / n_{0}$, the results for the two phases approach the thermal value, as required thermodynamically. At intermediate fields, however, we observe for a given field that the mean energy in the liquid phase is greater than that in the dilute gaseous phase indicating that energy can be deposited into the positron swarm more efficiently in the liquid phase. The reduction in the effective momentum transfer cross section at a given energy brought about by coherent scattering processes reduces the randomization in direction due to collisions and consequently facilitates enhanced power input from the field. The rapid increase in the mean energy appears as the positrons experience the Ramsauer minimum. The plateau region at higher reduced fields is a reflection initially of rapidly increasing momentum transfer, followed by enhanced energy deposition into excitation and ionization channels and the preferential loss of higher energy positrons to Ps formation, so-called Psinduced cooling.

In Fig. 3, a comparison of drift velocities (flux and bulk) for positrons in liquid and dilute gaseous $\mathrm{Ar}$ is presented. In the low-field limit, the liquid phase drift velocity is more than an order of magnitude greater than those in the dilute gas phase. This reflects the reduction in the momentum transfer collision cross section (and higher order cross sections) due to coherent elastic scattering which consequently enhances the directed motion (and hence drift

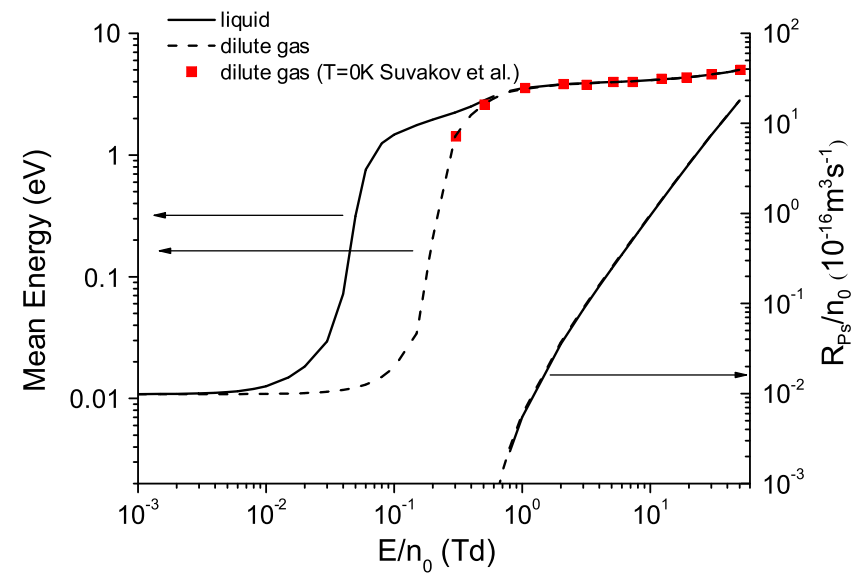

FIG. 2 (color online). The mean energy and Ps-formation rates for positrons in liquid and dilute gaseous $\mathrm{Ar}$ at $85 \mathrm{~K}$ as a function of $E / n_{0}$. These results are compared with the dilute gas phase results using Monte Carlo simulation [6]. velocity) of the positron swarm. At higher fields, Ps formation becomes significant (see Fig. 2), and the bulk and flux drift velocities diverge as the center of mass of the positron swarm is now explicitly modified through positron loss to Ps formation. These differences between the two types of drift velocity can be greater than 2 orders of magnitude. The most striking observation in Fig. 3, however, is the existence of two independent forms of NDC for positrons in liquid Ar. This contrasts the case for positrons in dilute gaseous Ar where only one form of NDC appears [6].

We will focus initially on the low-field NDC region present only in the liquid phase. In this field range there is limited excitation, ionization, or Ps formation (see Fig. 2), and it follows that this form of NDC is induced purely through the inclusion of the liquid structure, i.e., coherent elastic scattering. This form of NDC is referred to as "structure-induced NDC." In contrast to other forms of NDC for electron transport in dilute gases $[18,19]$, this form of NDC requires neither inelastic scattering processes nor nonconservative processes to induce it. To understand the origin of this effect and formulate conditions for its existence, we now consider the static spatially uniform momentum and energy balance equations formed from (1) using the momentum transfer approximation [16]:

$$
\begin{gathered}
e \boldsymbol{E}=\tilde{\nu}_{m}(\varepsilon) \boldsymbol{W}_{F}, \\
e \boldsymbol{E} \cdot \boldsymbol{W}_{F}=2 \frac{m}{m_{0}} \nu_{m}(\varepsilon)\left\{\varepsilon-\frac{3}{2} k T_{0}\right\},
\end{gathered}
$$

where $\nu_{m}=n_{0} c \sigma_{0}$ and $\tilde{\nu}_{m}=n_{0} c \Sigma_{m}=\nu_{m}\left(s_{0}-s_{1}\right)$. By using these relations, it can be shown that the condition for structure-induced NDC $\left(d W_{F} / d E<0\right)$ is $\frac{d \ln \left(s_{0}-s_{1}\right)}{d \ln \varepsilon}>1$ when coherent elastic scattering processes only are operative. This criterion is independent of the energy depen-

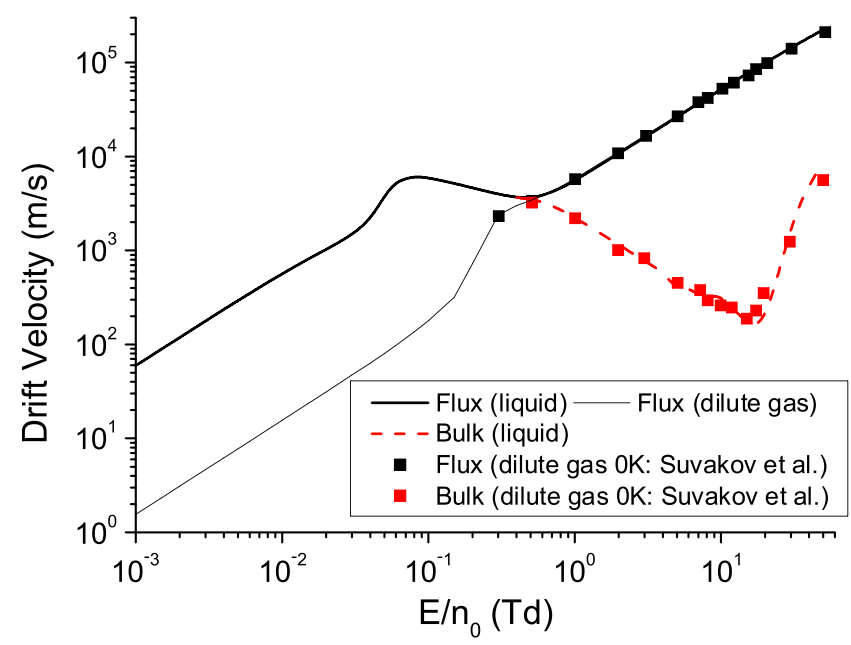

FIG. 3 (color online). The flux and bulk drift velocity of positrons in liquid and dilute gaseous $\mathrm{Ar}$ at $85 \mathrm{~K}$ as a function $E / n_{0}$. These results are compared with the dilute gas phase results using Monte Carlo simulation [6]. 


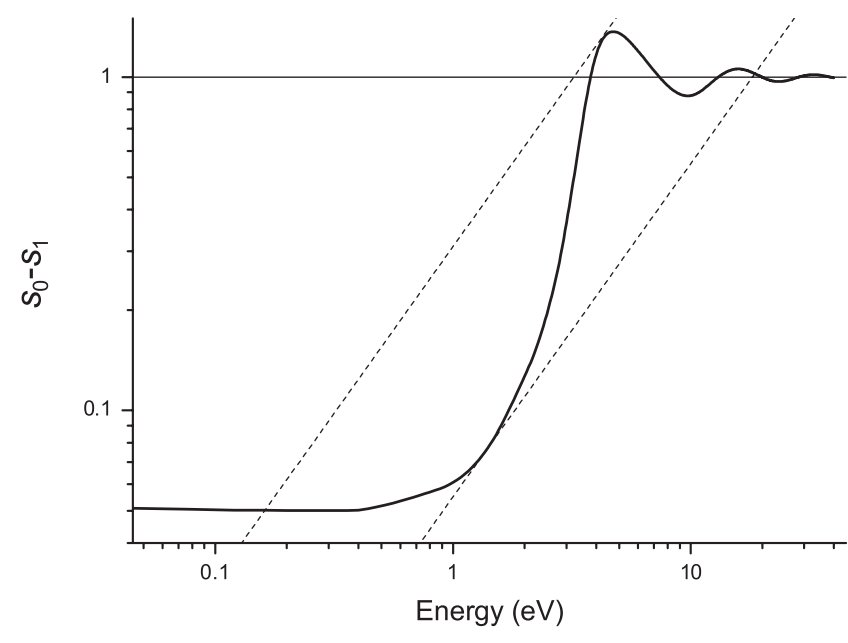

FIG. 4. A plot of the static structure factor moment $s_{0}-s_{1}$ as a function of energy. The dashed curves represent the critical lines for structure-induced NDC: $d \ln \left(s_{0}-s_{1}\right) / d \ln \epsilon=1$.

dence of the scattering cross sections and is purely dependent on the energy variation of the moments of $S$. In Fig. 4, we plot $s_{0}-s_{1}$ as a function of $\varepsilon$ and include the critical lines for NDC. By using Fig. 2, the lower bound on the structure-induced NDC condition is consistent with the onset on structure-induced NDC in Fig. 3. The upper bound is overestimated by the criterion, however, as in this region inelastic and Ps-formation processes are significant. We could modify the condition to include these additional effects, but such details are beyond the scope of this Letter. In the dilute gaseous case, $s_{0}-s_{1}$ is unity and hence structure-induced NDC does not arise. This criterion also carries over to electron transport in liquids and dense gas systems.

Moving to higher values of $E / n_{0}$, we see that $W$ and $W_{F}$ diverge due to the onset of Ps formation, and moreover a second form of NDC emerges in bulk drift velocity $W$ only, viz., Ps-formation-induced NDC. Its origin is distinctly different from structure-induced NDC discussed above and is brought about purely by positron loss associated with Ps formation, for reasons similar to that given by [6] in a Monte Carlo analysis of the gas phase. The present study, based on kinetic theory, demonstrates that Ps-formation-induced NDC carries over to the liquid phase. As for gases, the effect is basically due to the preferential depletion of more energetic positrons in Ps formation, and the reader is referred to [6] for a detailed explanation.

Concluding remarks.-We have presented the first multiterm solution of a new kinetic equation describing positron transport in soft condensed matter. The kinetic equation includes coherent elastic scattering (via the generalization of the Cohen and Lekner collision operator), excitation and ionization scattering, and Ps formation. The kinetic equation and associated solution technique are general and can be applied in the continuum from the dilute gas phase through to the liquid phase. The technique has been applied for the first time to positrons in liquid and dilute gaseous argon at $85 \mathrm{~K}$. We find that transport properties of positrons in liquids approach the dilute gaseous values in the high field limit. Of particular note is that the existence of two different sources of NDC are present for positrons in liquid Ar: (i) structure-induced NDC brought about by coherent elastic scattering (which is absent in the dilute gas phase limit), and (ii) Ps-induced NDC (which is also present in dilute gaseous phase). Such phenomena may be measurable through suitable adaptation of the experimental techniques detailed in $[14,20]$. Future investigations will aim to identify other multiscattering and localization effects that have been present for electrons in liquids.

The authors acknowledge the support of the Australian Research Council and Professor Petrović, staff, and students at the Institute of Physics, Belgrade for fruitful discussions.

*Ronald.White@jcu.edu.au

[1] S. R. Cherry, J. A. Sorensen, and M. E. Phelps, Physics in Nuclear Medicine (Saunders, Philadelphia, 2003).

[2] C. M. Surko, G. F. Gribakin, and S. J. Buckman, J. Phys. B 38, R57 (2005).

[3] A.S. Kadyrov, I. Bray, and A. T. Stelbovics, Phys. Rev. Lett. 98, 263202 (2007).

[4] J. Mitroy and M. W. J. Bromley, Phys. Rev. Lett. 98, 173001 (2007).

[5] A. Zecca et al., J. Phys. B 41, 085201 (2008).

[6] M. Suvakov et al., New J. Phys. 10, 053034 (2008).

[7] A. Bankovic et al., Nucl. Instrum. Methods Phys. Res., Sect. B 266, 462 (2008).

[8] Y. Sakai, J. Phys. D 40, R441 (2007).

[9] M. H. Cohen and J. Lekner, Phys. Rev. 158, 305 (1967).

[10] C. S. Wang-Chang, G. E. Uhlenbeck, and J. De Boer, in Studies in Statistical Mechanics, edited by J. D. Boer and G. E. Uhlenbeck (Wiley, New York, 1964), Vol. II, p. 241.

[11] K.F. Ness and R.E. Robson, Phys. Rev. A 34, 2185 (1986).

[12] R.E. Robson and K.F. Ness, Phys. Rev. A 33, 2068 (1986).

[13] L. Van Hove, Phys. Rev. 95, 249 (1954).

[14] I. Pepe et al., J. Phys. B 28, 3643 (1995).

[15] H. Tagashira, Y. Sakai, and S. Sakamoto, J. Phys. D 10, 1051 (1977).

[16] R. E. Robson, Introductory Transport Theory for Charged Particles in Gases (World Scientific, Singapore, 2006).

[17] J. L. Yarnell et al., Phys. Rev. A 7, 2130 (1973).

[18] Z. Lj. Petrovic, R. W. Crompton, and G. N. Haddad, Aust. J. Phys. 37, 23 (1984).

[19] S. B. Vrhovac and Z. Lj. Petrović, Phys. Rev. E 53, 4012 (1996).

[20] M. Charlton and J.W. Humberston, Positron Physics (Cambridge University Press, Cambridge, England, 2001). 